\title{
ELASTODYNAMICS OF A PARALLEL SCHÖNFLIES-MOTION GENERATOR
}

\author{
Zuyu Yin ${ }^{1}$, Bruno Belzile ${ }^{1}$, Jorge Angeles ${ }^{1}$, James Richard Forbes ${ }^{1}$ \\ ${ }^{1}$ Centre for Intelligent Machines and Department of Mechanical Engineering, McGill University, Montreal, Quebec, \\ Canada
}

Email:zuyuyin@cim.mcgill.ca;bruno@cim.mcgill.ca;angeles@cim.mcgill.ca;james.richard.forbes@mcgill.ca

Corresponding author:

Bruno Belzile

17 rue Sherbrooke Ouest room 270, Montréal, QC H3A 0C3

bruno@cim.mcgill.ca

\section{ÉLASTODYNAMIQUE D’UN GÉNÉRATEUR PARALLÈLE DE MOUVEMENTS DE SCHÖNFLIES}




\section{ABSTRACT}

The authors propose a model of the elastodynamics of the Peppermill Carrier (PMC), a parallel isostatic Schönflies-motion generator designed for pick-and-place operations. The Cartesian spring and the finite element method are used to build the elastodynamics model of the robot. The stiffness and mass matrices are introduced to obtain the natural frequencies of the robot along a test trajectory - the Adept test cyclethat serves to evaluate the performance of the robot with respect to the operation speed.

Keywords: Elastostatics; elastodynamics; Schönflies-motion generator.

\section{RÉSUMÉ}

Les auteurs proposent un modèle élastodynamique du Peppermill Carrier, un générateur isostatique parallèle de mouvements de Schönflies conçu pour les opérations de transfert. Le concept du ressort cartésien et la méthode des éléments finis sont utilisés pour obtenir le modèle élastodynamique du robot. Les matrices de raideur et de masse sont déterminées pour obtenir les fréquences naturelles du robot le long d'une trajectoire test-Adept test cycle—ce qui permet d'évaluer la performance du robot en termes de vitesse d'opération.

Mots-clés : Élastostatique; élastodynamique; générateur de mouvements de Schönflies. 


\section{INTRODUCTION}

Pick-and-place operations (PPOs) pertain to tasks that involve the moving of an object from one location to another. To produce these operations, serial and parallel robots are commonly used. While the former are known to have large workspaces with respect to their footprint, dexterous capabilities and ease of control, the latter offer many advantages in terms of speed, accuracy, dynamic response, load-carrying capacity, and stiffness. The Selective Compliance Assembly Robot Arm (SCARA) is the first and best-known example of a serial pick-and-place robot (Makino et al. 2007). The set of motions produced by pick-and-place robots is known to form a subgroup, the Schönflies subgroup, of the Lie group of rigid-body motions (Schoenflies 1893). Systems implementing these motions are designed with four degrees of freedom (dof), namely three independent translations and one rotation about one axis of fixed orientation.

The H4 robot (Pierrot and Company 1999), a parallel Schönflies-motion generator (SMG), first proposed by a French-Japanese team, was patented in 2001 (Pierrot et al. 2003). It consists of one moving platform, one base platform and four identical limbs. A detailed review on the structural synthesis of SMG was published by Gogu (2007), who claimed that there are totally three kinds of methods used for the structural synthesis of SMG, based on: displacement group theory (Angeles 2004, Hervé 2004, Li et al. 2004); screw algebra (Carricato 2005, Frisoli et al. 2000, Kong and Gosselin 2004); and the theory of linear transformations (Gogu 2004, 2005).

Commonly used, four-limb SMG architectures are plagued with limb interference, which results in limited rotation capability, of less than $180^{\circ}$ without the use of complex mechanisms inside the moving platform (MP). In the last two decades, many attractive four-limb parallel architectures were proposed, such as H4 (Pierrot and Company 1999), I4L (Company et al. 2002), I4R (Krut et al. 2004), Heli4 (Krut et al. 2006) and PAR4 (Nabat et al. 2005). An improved version of PAR4 became the Adept Quattro robot (Pierrot et al. 2009), which is the fastest parallel robot nowadays.

Compared with four-limb SMGs, two-limb SMGs have smaller footprint and virtually unlimited rotational displacement of the MP, but their stiffness is reduced (Gauthier et al. 2009). Two-limb isoconstrained architectures have many advantages, such as more compact envelope, ease of control and low sensitivity to manufacturing errors (Lee and Lee 2016). Mechanical isotopy ${ }^{2}$ improves the robustness of kinetostatic,

\footnotetext{
${ }^{2}$ Isotropy, associated with the condition number of the robot Jacobian matrix (Salisbury and Craig 1982), offers the maximum dexterity at a given robot posture.
} 
elastostatic and elastodynamic performance (Al-Widyan and Angeles 2004). Harada and Angeles studied an isoconstrained two-limb SMG, dubbed the Peppermill Carrier (PMC) (Harada and Angeles 2014), featuring an architecture proposed by the Lees (Lee and Lee 2012, Lee et al. 2010). The PMC is driven by two identical cylindrical drives (C-drives) (Harada et al. 2014). The C-drive design is based on a differential mechanism generator of the cylindrical subgroup; it produces both a rotation and an independent translation in the direction of the axis of rotation. A translating П-joint with a strain-wave-gear (SWG) drive was proposed to enhance the load-carrying capacity of the C-drive (Karimi Eskandary and Angeles 2018) and validated experimentally with an optimum gear-reduction ratio (Belzile et al. 2020). Velocity scheduling algorithms with time-scaling functions were also proposed to increase the attainable operation frequency of the PMC (Belzile and Angeles 2019, Karimi Eskandary et al. 2019). While the objective is to build a manipulator as stiff as possible, deformations are unavoidable due to inertia forces brought about by high-speed/high-acceleration operations. Therefore, manipulators must be considered flexible under these circumstances. Flexible-link manipulators have been applied in many scientific realms, involving microsurgical devices (Kumar et al. 2000), vibration (Dubowsky 1994) and launch cost reduction (Yamano et al. 2000) in astronautics, high-precision PPOs in industry (Shaheed et al. 2005) and pollution decontamination in nuclear sites (Meggiolaro and Dubowsky 2001). However, flexible components of a parallel manipulator will bring about vibration when fast PPOs are conducted. Therefore, the residual vibration after the motion stops will make the settling time longer and positioning accuracy lower. Moreover, vibration will impact on system stability (Zhang et al. 2016).

In this paper, the elastostatics of the PMC is first studied. Then, the trajectory used to obtain numerical data, the Adept test cycle, is briefly detailed. Finally, the elastodynamics, including a Fourier analysis, is conducted, the ensuing results then being discussed to assess the stiffness of the PMC. This work is an enhanced version of a paper presented at the 2019 CCToMM Symposium (Yin et al. 2019).

\section{ELASTOSTATICS}

\subsection{Model}

The hypothesis underlying the elastostatics model of the PMC is summarized as: all links are modelled as rigid bodies, except for the arms and forearms, as illustrated in Figs. 1 and 2. The reason for this hypothesis lies in that the latter are significantly more flexible than the other links. Notice that the screws of the C-drives 
and those of the Peppermill cannot be assumed flexible, because a screw joint, just like a prismatic joint, will jam if the joint deforms. Moreover, the flexibility of the strain-wave-gear drive is taken into consideration. While it is not required, the arms and forearms of the PMC have the same length. The limbs of the PMC and the SWG-enhanced C-drive are depicted in Figs. 3 and 4. The kinematic chain of the PMC is shown

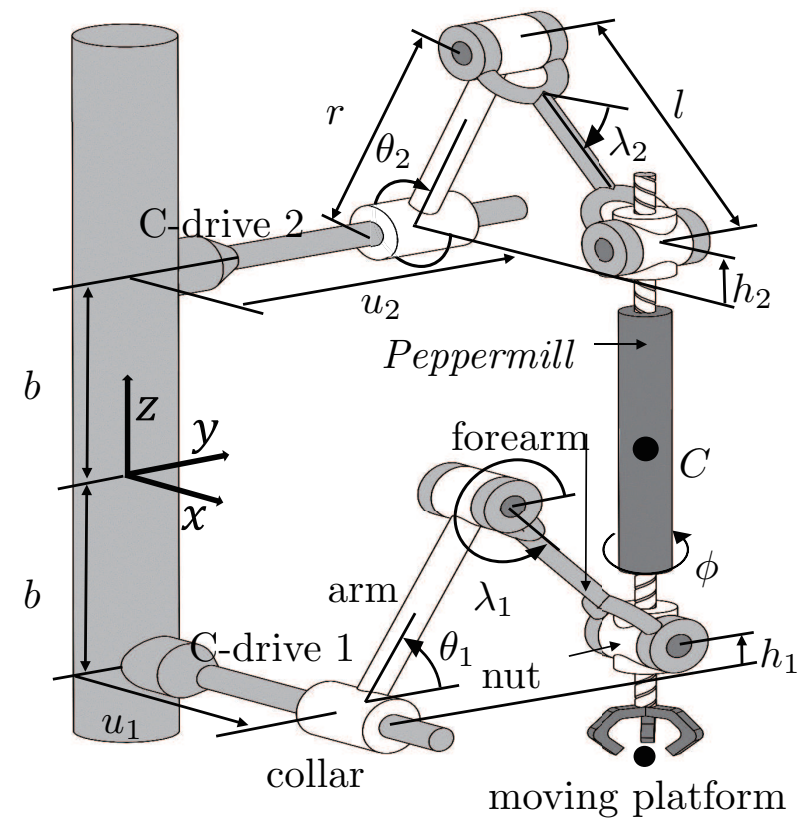

Fig. 1. Kinematics of the PMC

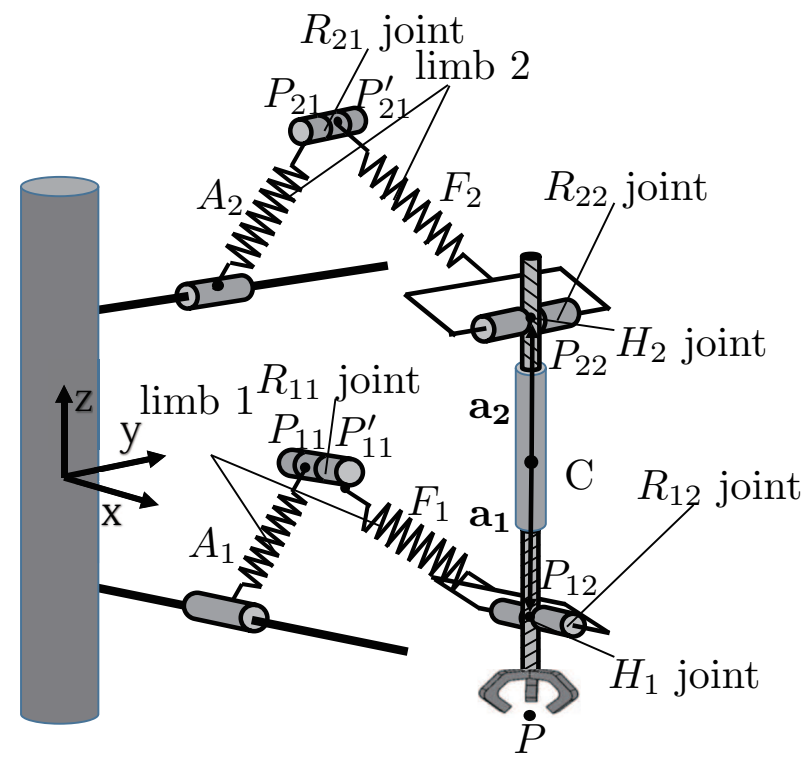

Fig. 2. Elastostatics of the PMC

in Fig. 1. The robot is modelled as an elastostatic system, like the one illustrated in Fig. 2. Each of the four 
springs of the figure is, in fact, a Cartesian spring, as defined by Lončarić (1985), i.e., a lump of massless, linearly elastic material mounted on a rigid plate and supporting a rigid body on top, as illustrated in Fig. 5.

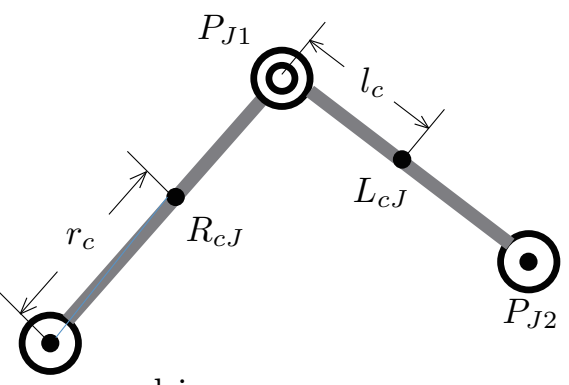

strain-wave-gear drive

Fig. 3. Side view of limb $J$

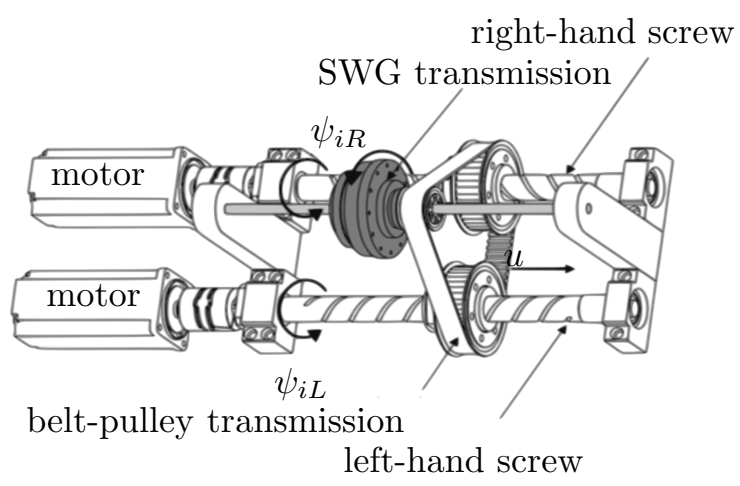

Fig. 4. SWG-driven C-drive

The two arms and the two forearms are thus modelled as Cartesian springs. As the rigid body on top of the Cartesian spring is acted upon by an external wrench, the body undergoes a small-amplitude displacement, the Cartesian spring then responding with a balancing wrench, identical to the applied wrench, but of opposite sign. Let $\mathbf{q}_{J 1}$ be the small-amplitude-displacement (SAD) screw defined at point $P_{J 1}$, where $J$ is the limb label, for $J=1,2$, as depicted in Fig. 2. Furthermore, let $\mathbf{q}_{J 2}$ be the SAD screw defined at point $P_{J 2}$. By virtue of the presence of the $R_{J 1}$ joint, the $\mathrm{SAD}$ screw, defined at point $P_{J 1}^{\prime}$, becomes

$$
\mathbf{q}_{J 1}^{\prime}=\mathbf{q}_{J 1}+\delta \beta_{J} \mathbf{s}_{J}, \quad \mathbf{s}_{J}=\left[\begin{array}{ll}
\mathbf{e}_{J}^{T} & \mathbf{0}^{T}
\end{array}\right]^{T}
$$

where $\mathbf{e}_{J}$ is the unit vector parallel to the axis of the $R_{J 1}$ joint and $\delta \beta_{J}$ the small-amplitude relative rotation about the same axis. The forearms are connected to the nuts via $\mathrm{R}$ (revolute) joints of horizontal axes. The SAD screws defined at the centre of mass $(\mathrm{COM})$ of the nuts $\left(P_{J 2}\right)$ and the COM of the Peppermill $(C)$ are 


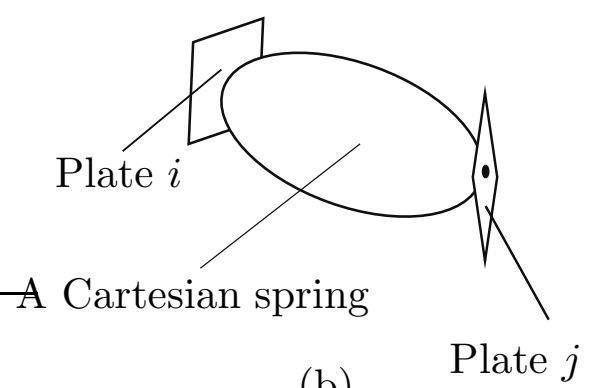

(b)

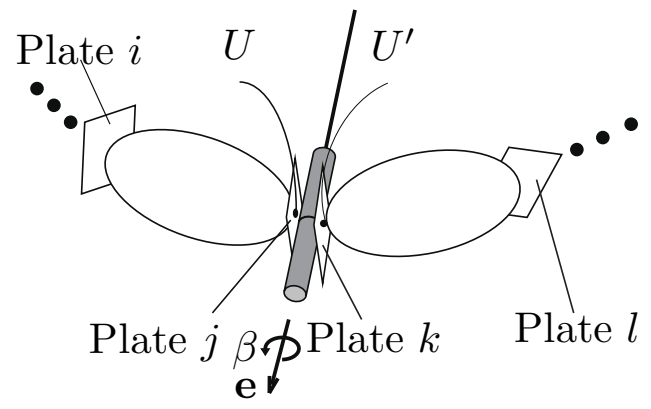

(a)

Fig. 5. The concept of Cartesian spring: (a) two rigid plates coupled by a Cartesian spring; (b) the coupling of two Cartesian springs via a $\mathrm{R}$ joint

represented by $\mathbf{q}_{n J}$ and $\mathbf{q}_{m}$, respectively. According to the rigidity assumption and the presence of the $\mathrm{R}$ joint, the relationship between $\mathbf{q}_{J 2}$ and $\mathbf{q}_{n J}$ is

$$
\mathbf{q}_{J 2}=\mathbf{q}_{n J}+\delta \gamma_{J} \mathbf{S}_{J}
$$

where $\delta \gamma_{J}$ is the "small" angle of rotation of the $R_{J 2}$ joint, and $\mathbf{s}_{J}$ is defined in eq. (1). The nuts are connected to the Peppermill via $\mathrm{H}$ joints of nominally vertical axes. The relationship between $\mathbf{q}_{n J}$ and $\mathbf{q}_{m}$ is

$$
\mathbf{q}_{n J}=\mathbf{G}_{J} \mathbf{q}_{m}+\delta \alpha_{J} \mathbf{s}_{H J}, \quad \mathbf{s}_{H J}=\left[\begin{array}{ll}
\mathbf{e}_{H J}^{T} & p_{J} \mathbf{e}_{H J}^{T}
\end{array}\right]^{T}
$$

where $\delta \alpha_{J}$ is the "small" angle of rotation of the $H_{J}$ joint with respect to the direction of its axis, $\mathbf{e}_{H J}$ the unit vector of its axis and $p_{J}$ the pitch of the $H_{J}$ joint. Moreover, $\mathbf{G}_{J}$ is the SAD screw transfer matrix (Pradeep et al. 1989) that takes the SAD screw of one given rigid body from one point to another point of the same body. In the case at hand, from point $C$ to point $P_{J 2}$ of the Peppermill, $\mathbf{G}_{J}$ being given by 


$$
\mathbf{G}_{J}=\left[\begin{array}{cc}
\mathbf{1} & \mathbf{0} \\
-\mathbf{A}_{J} & \mathbf{1}
\end{array}\right]
$$

where $\mathbf{A}_{J}=\operatorname{CPM}\left(\mathbf{a}_{J}\right)$, is the cross-product matrix of vector ${ }^{3} \mathbf{a}_{J}$, stemming from $C$ and ending at $P_{J 2}$.

\subsection{The Cartesian Stiffness Matrix}

The objective of elastostatic analysis is to obtain the Cartesian stiffness matrix $\mathbf{K}_{e}$ needed to conduct the modal analysis of the PMC. Matrix $\mathbf{K}_{e} \in \mathbf{R}^{6 \times 6}$ maps the SAD screw of the Peppermill into the external wrench applied onto it, which is given by

$$
\mathbf{w}_{\mathrm{ext}}=\mathbf{K}_{e} \mathbf{q}
$$

where $\mathbf{w}_{\mathrm{ext}}$ is the external wrench and $\mathbf{q}$ the SAD screw of the Peppermill. If we apply a unit external wrench on six different directions separately, the corresponding SAD screws are nothing but the columns of the inverse matrix of the Cartesian stiffness matrix.

Firstly, a unit external force in the $x$-direction, $\mathbf{w}_{f x}$, is applied at the centre of mass $C$ of the Peppermill. From the mechanical structure of the PMC, we can find that $\mathbf{w}_{f x}$ will bring about a deformation of the arm and the forearm of limbs 1 and 2. Through the force analysis of the Peppermill, balancing forces $\mathbf{f}_{f 1 p}$ and $\mathbf{f}_{f 2 p}$ are added on the Peppermill at points $P_{12}$ and $P_{22}$, respectively. Since the effects of force are mutual, reactive forces $\mathbf{f}_{p f 1}=-\mathbf{f}_{f 1 p}$ and $\mathbf{f}_{p f 2}=-\mathbf{f}_{f 2 p}$ are applied on the forearm of each of limbs 1 and 2 , henceforth termed forearm 1 and forearm 2. Therefore, we can obtain the SAD screws of the two forearms in the forms

$$
\mathbf{q}_{f 1}=\left(\mathbf{K}^{F 1}\right)^{-1} \mathbf{w}_{p f 1}, \quad \mathbf{q}_{f 2}=\left(\mathbf{K}^{F 2}\right)^{-1} \mathbf{w}_{p f 2}
$$

where $\mathbf{K}^{F 1}$ and $\mathbf{K}^{F 2}$ denote the Cartesian stiffness matrices of forearm 1 and forearm 2, respectively, while $\mathbf{w}_{p f 1}=\left[\begin{array}{ll}\mathbf{m}_{p f 1}^{T} & \mathbf{f}_{p f 1}^{T}\end{array}\right]^{T}$ and $\mathbf{w}_{p f 2}=\left[\begin{array}{ll}\mathbf{m}_{p f 2}^{T} & \mathbf{f}_{p f 2}^{T}\end{array}\right]^{T}$. On the other hand, the forces acting on forearm 1 and forearm 2 will be transferred to arm 1 and arm 2 via corresponding passive revolute joints. The SAD screws of arm 1 and arm 2 are given by

$$
\mathbf{q}_{a 1}=\left(\mathbf{K}^{A 1}\right)^{-1} \mathbf{w}_{f 1 a 1}, \quad \mathbf{q}_{a 2}=\left(\mathbf{K}^{A 2}\right)^{-1} \mathbf{w}_{f 2 a 2}
$$

\footnotetext{
${ }^{3}$ The CPM of $\boldsymbol{A}_{J}$ is defined as the partial derivative of $\boldsymbol{A}_{J} \times \mathbf{y}$ with respect to $\mathbf{y}, \forall \mathbf{y} \in \mathbf{R}^{3}$.
} 
where $\mathbf{K}^{A J}$ denotes the Cartesian stiffness matrix of arm $J$, while $\mathbf{w}_{f 1 a 1}=\mathbf{w}_{p f 1}$ and $\mathbf{w}_{f 2 a 2}=\mathbf{w}_{p f 2}$. The concept of SAD-screw transfer matrix is now recalled. Let $\mathbf{G}_{J c}$ be given by

$$
\mathbf{G}_{J c}=\left[\begin{array}{cc}
\mathbf{1} & \mathbf{0} \\
-\mathbf{A}_{J c} & \mathbf{1}
\end{array}\right]
$$

where $\mathbf{A}_{J c}=\operatorname{CPM}\left(\mathbf{a}_{J c}\right)$, vector $\mathbf{a}_{J c}$ stemming from $P_{J 2}$ and ending at $C . \mathbf{G}_{J c}$ transfers the SAD screw of point $P_{J 2}$ to point $C$, the COM of the Peppermill. Therefore, the total deformation caused by the external wrench $\mathbf{w}_{f x}$ is

$$
\mathbf{q}_{w f x}=\mathbf{G}_{1 c}\left(\mathbf{q}_{a 1}+\mathbf{q}_{f 1}\right)+\mathbf{G}_{2 c}\left(\mathbf{q}_{a 2}+\mathbf{q}_{f 2}\right)
$$

The SAD screws $\mathbf{q}_{w f y}, \mathbf{q}_{w m x}, \mathbf{q}_{w m y}$ and $\mathbf{q}_{w m z}$, produced by the unit external wrenches $\mathbf{w}_{f y}, \mathbf{w}_{m x}, \mathbf{w}_{m y}$ and $\mathbf{w}_{m z}$ can be obtained likewise. The deformation $\mathbf{q}_{w f z}$, caused by the unit external force in the $z$-direction, will be analyzed separately because it is related to the flexibility of the strain-wave-gear drive.

A unit external force $\mathbf{w}_{f z}$ is applied at the centre of mass $C$ of the Peppermill in the $z$-direction. Because of the symmetric mechanical structure, each of the wrenches acting on the Peppermill by forearm 1, $\mathbf{w}_{f 1 p}$, and forearm 2, $\mathbf{w}_{f 2 p}$, equals half of $\mathbf{w}_{f z}$. Therefore, the deformation of forearm $J$ is given by

$$
\mathbf{q}_{f J}=\left(\mathbf{K}^{F J}\right)^{-1} \mathbf{w}_{p f J}, \quad J=1,2
$$

where $\mathbf{w}_{p f J}=-\mathbf{w}_{f J p}$. On the other hand, the deformation of $\operatorname{arm} J$ is given by

$$
\mathbf{q}_{a J}=\left(\mathbf{K}^{A J}\right)^{-1} \mathbf{w}_{f J a J}, \quad J=1,2
$$

where $\mathbf{w}_{f J a J}=\mathbf{w}_{p f J}$. As for the angular displacements of the strain-wave-gear drives, which are given by

$$
\alpha_{J}=f_{f J a J} \cos \left(\theta_{J}\right) r / k_{\text {harm }}, \quad J=1,2
$$

where $f_{f 1 a 1}$ and $f_{f 2 a 2}$ are the force components of $\mathbf{w}_{f 1 a 1}$ and $\mathbf{w}_{f 2 a 2}$ in the $z$-direction, $\theta_{1}$ and $\theta_{2}$ defined in Fig. $1, k_{\text {harm }}$ the torsional stiffness of the strain-wave-gear drive. Therefore, the deformation caused by the 
strain-wave-gear drives at points $P_{11}$ and $P_{21}$ is

$$
\mathbf{q}_{h J}=\left[\begin{array}{ll}
\mathbf{0}^{T} & \mathbf{d}_{h J}^{T}
\end{array}\right]^{T}, \quad \mathbf{d}_{h J}=\left[\begin{array}{lll}
(J-1) \alpha_{2} r \sin \left(\theta_{2}\right) & (J-2) \alpha_{J} r \sin \left(\theta_{J}\right) & \alpha_{J} \cos \left(\theta_{J}\right)
\end{array}\right]^{T}, \quad J=1,2
$$

where $\mathbf{0}$ is the three-dimensional zero vector. The deformation caused by the unit external wrench $\mathbf{w}_{f z}$ is

$$
\mathbf{q}_{w f z}=\mathbf{G}_{1 c}\left(\mathbf{q}_{a 1}+\mathbf{q}_{f 1}+\mathbf{q}_{h 1}\right)+\mathbf{G}_{2 c}\left(\mathbf{q}_{a 2}+\mathbf{q}_{f 2}+\mathbf{q}_{h 2}\right)
$$

Since the unit external forces and moments are applied at the COM of the Peppermill, the SAD screws are nothing but the columns of the inverse matrix of the Cartesian stiffness matrix of the PMC. Therefore, matrix $\mathbf{K}_{e}$ is given by

$$
\mathbf{K}_{e}=\left[\begin{array}{llllll}
\mathbf{q}_{w m x} & \mathbf{q}_{w m y} & \mathbf{q}_{w m z} & \mathbf{q}_{w f x} & \mathbf{q}_{w f y} & \mathbf{q}_{w f z}
\end{array}\right]^{-1}
$$

In the above analysis, $\mathbf{K}^{A J}$ and $\mathbf{K}^{F J}$ are defined in the base frame and $\overline{\mathbf{K}}^{A J}$ and $\overline{\mathbf{K}}^{F J}$ denote the stiffness matrices of arms and forearms defined in the body-fixed frame. $\overline{\mathbf{K}}^{A J}$ and $\overline{\mathbf{K}}^{F J}$ are posture-independent, obtained by FEA. $\mathbf{K}^{A J}$ and $\mathbf{K}^{F J}$ are posture-dependent, derived from $\overline{\mathbf{K}}^{A J}, \overline{\mathbf{K}}^{F J}$ by means of similarity transformations in terms of $6 \times 6$ rotation matrices, as described below. The coordinate frames of the arms and the forearms are shown in Fig. 6. $\mathscr{F} 0$ represents the fixed frame. $\mathbf{Q}_{J 10}$ is the rotation matrix that carries $\mathscr{F}_{J 1}$ into $\mathscr{F}_{0}$. Similarly, $\mathbf{Q}_{J 21}$ and $\mathbf{Q}_{J 20}$ are the rotation matrices that carry $\mathscr{F}_{J 2}$ into $\mathscr{F}_{J 1}$ and $\mathscr{F}_{J 2}$ into $\mathscr{F}_{0}$, respectively. A $6 \times 6$ rotation matrix $\mathbf{R}_{J 10}$ is now introduced to transfer six-dimensional SAD screws from $\mathscr{F}_{J 1}$ into $\mathscr{F}_{0}$ :

$$
\mathbf{R}_{J 10}=\left[\begin{array}{cc}
\mathbf{Q}_{J 10} & \mathbf{O} \\
\mathbf{O} & \mathbf{Q}_{J 10}
\end{array}\right]
$$

Similarly, the $6 \times 6$ rotation matrices $\mathbf{R}_{J 21}$ and $\mathbf{R}_{J 20}$ are further introduced:

$$
\mathbf{R}_{J 21}=\left[\begin{array}{cc}
\mathbf{Q}_{J 21} & \mathbf{O} \\
\mathbf{O} & \mathbf{Q}_{J 21}
\end{array}\right], \quad \mathbf{R}_{J 20}=\left[\begin{array}{cc}
\mathbf{Q}_{J 20} & \mathbf{O} \\
\mathbf{O} & \mathbf{Q}_{J 20}
\end{array}\right]
$$

where $\mathbf{R}_{J 21}$ and $\mathbf{R}_{J 20}$ carry $\mathscr{F}_{J 2}$ into $\mathscr{F}_{J 1}$ and $\mathscr{F}_{J 2}$ into $\mathscr{F}_{0}$, respectively. Therefore, the relationships between 
$\mathbf{K}^{A J}\left(\mathbf{K}^{F J}\right)$ and its "overlined" counterpart $\overline{\mathbf{K}}^{A J}\left(\overline{\mathbf{K}}^{F J}\right)$ are readily derived:

$$
\mathbf{K}^{A J}=\mathbf{R}_{J 10}^{T} \overline{\mathbf{K}}^{A J} \mathbf{R}_{J 10}, \quad \mathbf{K}^{F J}=\mathbf{R}_{J 20}^{T} \overline{\mathbf{K}}^{F J} \mathbf{R}_{J 20}
$$

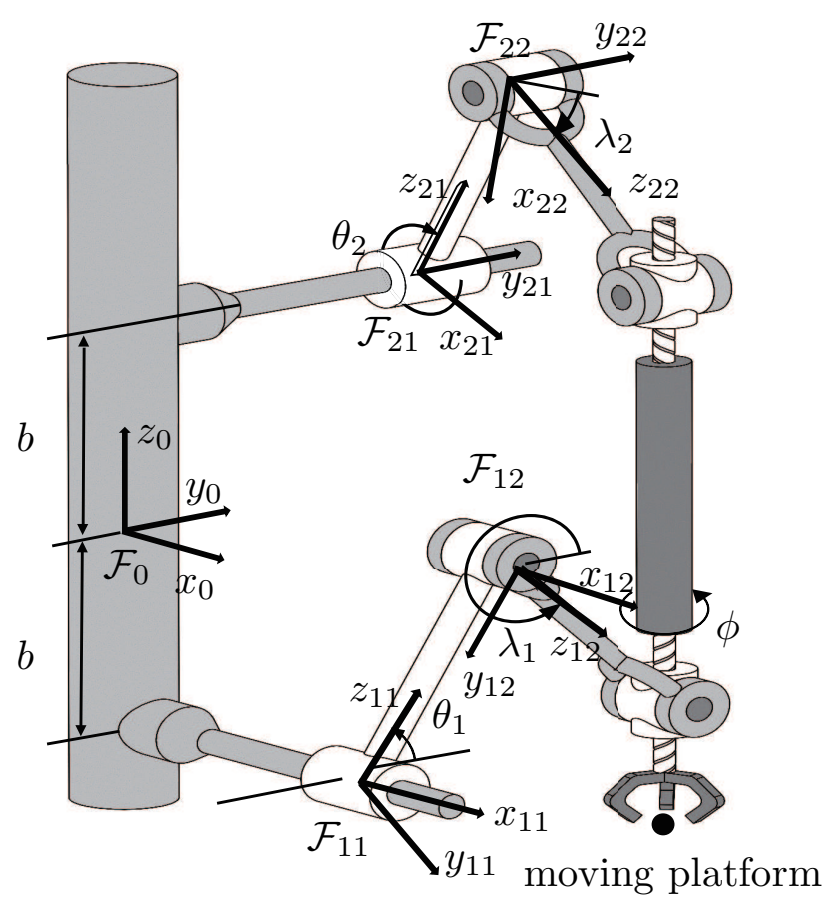

Fig. 6. Cartesian coordinates and body-fixed coordinates

\section{ELASTODYNAMICS}

To formulate the kinetic energy generated by the flexible-component motion, the generalized coordinates and the generalized velocities are defined below. The independent generalized-coordinate array is defined as

$$
\mathbf{q}=\left[\begin{array}{lllllllll}
\mathbf{q}_{m}^{T} & \mathbf{q}_{11}^{T} & \mathbf{q}_{21}^{T} & \delta \gamma_{1} & \delta \gamma_{2} & \delta \beta_{1} & \delta \beta_{2} & \delta \alpha_{1} & \delta \alpha_{2}
\end{array}\right]^{T}
$$

with the corresponding generalized velocity array $\dot{\mathbf{q}}$ following suit:

$$
\dot{\mathbf{q}}=\left[\begin{array}{lllllllll}
\dot{\mathbf{q}}_{m}^{T} & \dot{\mathbf{q}}_{11}^{T} & \dot{\mathbf{q}}_{21}^{T} & \delta \dot{\gamma}_{1} & \delta \dot{\gamma}_{2} & \delta \dot{\beta}_{1} & \delta \dot{\beta}_{2} & \delta \dot{\alpha}_{1} & \delta \dot{\alpha}_{2}
\end{array}\right]^{T}
$$


As defined in Subsection 2.1, the components of $\mathbf{q}$ are, respectively, the SAD screws defined at $C, P_{11}, P_{21}$, and the small-amplitude angle of rotation of the $R_{12}, R_{22}, R_{11}, R_{21}, H_{1}, H_{2}$ joints, Since all the motors are locked at a particular posture, the motion is generated by the deformation of the flexible components. As for the PMC, the kinetic energy is generated by the elastic motion of the arms, the forearms, the nuts and the Peppermill, all displayed in Fig. 1. Let, for $J=1,2, \dot{\mathbf{q}}_{J 1}, \dot{\mathbf{q}}_{J 1}^{\prime}, \dot{\mathbf{q}}_{J 2}, \dot{\mathbf{q}}_{n J}$ and $\dot{\mathbf{q}}_{m}$ denote the corresponding twists, defined at the points $P_{J 1}, P_{J 1}^{\prime}, P_{J 2}, P_{J 2}^{\prime}$ and $C$, respectively, as shown in Fig. 2.

As mentioned in Subsection 2.1, a schematic side view of limb $J$ is shown in Fig. 3, where the points $R_{c J}$ and $L_{c J}$ are the COM of the arm and the forearm, respectively. Let $\dot{\mathbf{q}}_{a J}$ and $\dot{\mathbf{q}}_{f J}$ denote the twists defined at points $R_{c J}$ and $L_{c J}$, respectively. The relationship between $\dot{\mathbf{q}}_{J 1}$ and $\dot{\mathbf{q}}_{a J}$ is

$$
\dot{\mathbf{q}}_{a J}=\mathbf{G}_{J 1} \dot{\mathbf{q}}_{J 1}, \quad \mathbf{G}_{J 1}=\left[\begin{array}{cc}
\mathbf{1} & \mathbf{0} \\
-\mathbf{R}_{J} & \mathbf{1}
\end{array}\right]
$$

where $\mathbf{R}_{J}=\mathrm{CPM}\left(\mathbf{r}_{c J}\right)$, vector $\mathbf{r}_{c J}$ stemming from $P_{J 1}$ and ending at $R_{c J}$. Similarly, the relationships among $\dot{\mathbf{q}}_{J 1}^{\prime}, \dot{\mathbf{q}}_{J 2}$ and $\dot{\mathbf{q}}_{f J}^{\prime}$ are readily derived:

$$
\dot{\mathbf{q}}_{f J}=\mathbf{G}_{J 2} \dot{\mathbf{q}}_{J 1}^{\prime}+\mathbf{G}_{J 3} \dot{\mathbf{q}}_{J 2}, \quad \mathbf{G}_{J 2}=\left[\begin{array}{rr}
\mathbf{1} & \mathbf{0} \\
-\mathbf{L}_{J} & \mathbf{1}
\end{array}\right], \quad \mathbf{G}_{J 3}=\left[\begin{array}{cc}
\mathbf{1} & \mathbf{0} \\
-\mathbf{L}_{J}^{\prime} & \mathbf{1}
\end{array}\right]
$$

where $\mathbf{L}_{J}=\mathrm{CPM}\left(\mathbf{l}_{c J}\right), \mathbf{L}_{J}^{\prime}=\mathrm{CPM}\left(\mathbf{l}_{c J}^{\prime}\right)$, vector $\mathbf{l}_{c J}$ stemming from $P_{J 1}^{\prime}$ and ending at $L_{c J}$, its primed counterpart, $\mathbf{I}_{c J}^{\prime}$, stemming from $P_{J 2}$ and ending at the same point $L_{c J}$. The kinetic energy of $\operatorname{arm} J$ is

$$
T_{J}^{A}=\frac{1}{2} \dot{\mathbf{q}}_{a J}^{T} \mathbf{M}^{A J} \dot{\mathbf{q}}_{a J}, \quad \mathbf{M}^{A J}=\left[\begin{array}{cc}
\mathbf{I}^{A J} & \mathbf{O} \\
\mathbf{O} & m^{A J} \mathbf{1}
\end{array}\right]
$$

where $\mathbf{M}^{A J}$, $\mathbf{I}^{A J}$ and $m^{A J}$ are the von Mises inertia dyad (Mises 1924), the inertia tensor and the mass of arm J, respectively. Similarly, the kinetic energy of the forearm, the nut and the Peppermill are further introduced:

$$
T_{J}^{F}=\frac{1}{2} \dot{\mathbf{q}}_{f J}^{T} \mathbf{M}^{F J} \dot{\mathbf{q}}_{f J}, \quad T_{J}^{N}=\frac{1}{2} \dot{\mathbf{q}}_{n J}^{T} \mathbf{M}^{N J} \dot{\mathbf{q}}_{n J}, \quad T^{P}=\frac{1}{2} \dot{\mathbf{q}}_{m}^{T} \mathbf{M}^{P} \dot{\mathbf{q}}_{m}
$$

where $\mathbf{M}^{F J}, \mathbf{M}^{N J}$ and $\mathbf{M}^{P}$ are the inertia dyads of: forearm and nut of limb $\mathrm{J}$, and of the Peppermill, 
respectively, given by

$$
\mathbf{M}^{F J}=\left[\begin{array}{cc}
\mathbf{I}^{F J} & \mathbf{O} \\
\mathbf{O} & m^{F J} \mathbf{1}
\end{array}\right], \quad \mathbf{M}^{N J}=\left[\begin{array}{cc}
\mathbf{I}^{N J} & \mathbf{O} \\
\mathbf{O} & m^{N J} \mathbf{1}
\end{array}\right], \quad \mathbf{M}^{P}=\left[\begin{array}{cc}
\mathbf{I}^{P} & \mathbf{O} \\
\mathbf{O} & m^{P} \mathbf{1}
\end{array}\right]
$$

The kinetic energy of the PMC is the sum of the kinetic energies of the forearms, the arms, the nuts and the Peppermill:

$$
T_{e}=\sum_{J=1}^{2}\left(T_{J}^{A}+T_{J}^{F}+T_{J}^{N}\right)+T^{P}
$$

Substitution of eqs. (21-24) into eq. (26), the expression for the kinetic energy of the PMC becomes,

$$
\begin{aligned}
T_{e}= & \frac{1}{2} \sum_{J=1}^{2}\left[\left(\mathbf{G}_{J 1} \dot{\mathbf{q}}_{J 1}\right)^{T} \mathbf{M}^{A J}\left(\mathbf{G}_{J 1} \dot{\mathbf{q}}_{J 1}\right)+\left(\mathbf{G}_{J 2} \dot{\mathbf{q}}_{J 1}^{\prime}+\mathbf{G}_{J 3} \dot{\mathbf{q}}_{J 2}\right)^{T} \mathbf{M}^{F J}\left(\mathbf{G}_{J 2} \dot{\mathbf{q}}_{J 1}^{\prime}+\mathbf{G}_{J 3} \dot{\mathbf{q}}_{J 2}\right)\right. \\
& \left.+\dot{\mathbf{q}}_{n J}^{T} \mathbf{M}^{N J} \dot{\mathbf{q}}_{n J}\right]+\frac{1}{2} \dot{\mathbf{q}}_{m}^{T} \mathbf{M}^{P} \dot{\mathbf{q}}_{m}
\end{aligned}
$$

Moreover, upon differentiation of the two sides of eq. (1), the relationship between $\dot{\mathbf{q}}_{J 1}$ and $\dot{\mathbf{q}}_{J 1}^{\prime}$ is obtained:

$$
\dot{\mathbf{q}}_{J 1}^{\prime}=\dot{\mathbf{q}}_{J 1}+\delta \dot{\beta}_{J} \mathbf{S}_{J}
$$

where, as mentioned above, $\delta \dot{\beta}_{J}$ is the the small-amplitude angular velocity about the $R_{J 1}$ joint, for $J=1,2$. By resorting to eqs. $(2 \& 3)$, the relationships between $\dot{\mathbf{q}}_{J 2}\left(\dot{\mathbf{q}}_{n J}\right)$ and its counterpart $\dot{\mathbf{q}}_{n J}\left(\dot{\mathbf{q}}_{m}\right)$ are readily derived:

$$
\dot{\mathbf{q}}_{J 2}=\dot{\mathbf{q}}_{n J}+\delta \dot{\gamma}_{J} \mathbf{S}_{J}, \quad \dot{\mathbf{q}}_{n J}=\mathbf{G}_{J} \dot{\mathbf{q}}_{m}+\delta \dot{\alpha}_{J} \mathbf{S}_{H J}
$$

where, as a reminder, $\delta \dot{\alpha}_{J}$ is the the small-amplitude angular velocity about the $H_{J}$ joint, for $J=1,2$. Substitution of eqs. (28 \& 29) into eq. (27), leads to

$$
\begin{aligned}
T_{e}= & \frac{1}{2} \sum_{J=1}^{2}\left[\left(\mathbf{G}_{J 1} \dot{\mathbf{q}}_{J 1}\right)^{T} \mathbf{M}^{A J}\left(\mathbf{G}_{J 1} \dot{\mathbf{q}}_{J 1}\right)+\mathbf{G}_{J 2}\left(\dot{\mathbf{q}}_{J 1}+\delta \dot{\beta}_{J} \mathbf{s}_{J}\right)\right. \\
& \left.+\mathbf{G}_{J 3}\left(\dot{\mathbf{q}}_{n J}+\delta \dot{\gamma}_{J} \mathbf{s}_{J}\right)\right)^{T} \mathbf{M}^{F J}\left(\mathbf{G}_{J 2}\left(\dot{\mathbf{q}}_{J 1}+\delta \dot{\beta}_{J} \mathbf{s}_{J}\right)+\mathbf{G}_{J 3}\left(\dot{\mathbf{q}}_{n J}+\delta \dot{\gamma}_{J} \mathbf{s}_{J}\right)\right. \\
& \left.+\left(\mathbf{G}_{J} \dot{\mathbf{q}}_{m}+\delta \dot{\alpha}_{J} \mathbf{s}_{H J}\right)^{T} \mathbf{M}^{N J}\left(\mathbf{G}_{J} \dot{\mathbf{q}}_{m}+\delta \dot{\alpha}_{J} \mathbf{s}_{H J}\right)\right]+\frac{1}{2} \dot{\mathbf{q}}_{m}^{T} \mathbf{M}^{P} \dot{\mathbf{q}}_{m}
\end{aligned}
$$

The generalized mass matrix of the PMC is the Hessian matrix of $T_{e}$ with respect to the generalized 
velocities. This matrix maps the generalized velocity array into the generalized momentum array:

$$
\left[\begin{array}{c}
\mathbf{p}_{m} \\
\mathbf{p}_{1} \\
\mathbf{p}_{2} \\
p_{12} \\
p_{22} \\
p_{11} \\
p_{21} \\
p_{13} \\
p_{23}
\end{array}\right]=\left[\begin{array}{ccccccccc}
\mathbf{M}_{11} & \mathbf{M}_{12} & \mathbf{M}_{13} & \mathbf{m}_{14} & \mathbf{m}_{15} & \mathbf{m}_{16} & \mathbf{m}_{17} & \mathbf{m}_{18} & \mathbf{m}_{19} \\
\mathbf{M}_{12}^{T} & \mathbf{M}_{22} & \mathbf{O} & \mathbf{m}_{24} & \mathbf{0} & \mathbf{m}_{26} & \mathbf{0} & \mathbf{m}_{28} & \mathbf{0} \\
\mathbf{M}_{13}^{T} & \mathbf{O} & \mathbf{M}_{33} & \mathbf{0} & \mathbf{m}_{35} & \mathbf{0} & \mathbf{m}_{37} & \mathbf{0} & \mathbf{m}_{39} \\
\mathbf{m}_{14}^{T} & \mathbf{m}_{24}^{T} & \mathbf{0}^{T} & m_{44} & 0 & m_{46} & 0 & m_{48} & 0 \\
\mathbf{m}_{15}^{T} & \mathbf{0}^{T} & \mathbf{m}_{35}^{T} & 0 & m_{55} & 0 & m_{57} & 0 & m_{59} \\
\mathbf{m}_{16}^{T} & \mathbf{m}_{26}^{T} & \mathbf{0}^{T} & m_{46} & 0 & m_{66} & 0 & m_{68} & 0 \\
\mathbf{m}_{17}^{T} & \mathbf{0}^{T} & \mathbf{m}_{37}^{T} & 0 & m_{57} & 0 & m_{77} & 0 & m_{79} \\
\mathbf{m}_{18}^{T} & \mathbf{m}_{28}^{T} & \mathbf{0}^{T} & m_{48} & 0 & m_{68} & 0 & m_{88} & 0 \\
\mathbf{m}_{19}^{T} & \mathbf{0}^{T} & \mathbf{m}_{39}^{T} & 0 & m_{59} & 0 & m_{79} & 0 & m_{99}
\end{array}\right]\left[\begin{array}{c}
\dot{\mathbf{q}}_{m} \\
\dot{\mathbf{q}}_{11} \\
\dot{\mathbf{q}}_{21} \\
\delta \dot{\gamma}_{1} \\
\delta \dot{\gamma}_{2} \\
\delta \dot{\beta}_{1} \\
\delta \dot{\beta}_{2} \\
\delta \dot{\alpha}_{1} \\
\delta \dot{\alpha}_{2}
\end{array}\right]
$$

where $\mathbf{p}_{m}, \mathbf{p}_{J}, p_{J 1}, p_{J 2}$ and $p_{J 3}$ are, respectively, the six-dimensional generalized momenta defined at $C$, $P_{J 1}$, and the generalized angular momenta about the $R_{J 1}, R_{J 2}$ and $H_{J}$ joints, $\mathbf{O}$ the $6 \times 6$ zero matrix, $\mathbf{0}$ the six-dimensional zero vector, the non-zero blocks of the mass matrix being described below:

$$
\begin{aligned}
& \mathbf{M}_{11}=\mathbf{G}_{1}^{T} \mathbf{G}_{13}^{T} \mathbf{M}^{F 1} \mathbf{G}_{13} \mathbf{G}_{1}+\mathbf{G}_{1}^{T} \mathbf{M}^{N 1} \mathbf{G}_{1}+\mathbf{G}_{2}^{T} \mathbf{G}_{23}^{T} \mathbf{M}^{F 2} \mathbf{G}_{23} \mathbf{G}_{2}+\mathbf{G}_{2}^{T} \mathbf{M}^{N 2} \mathbf{G}_{2}+\mathbf{M}^{P} \\
& \mathbf{M}_{12}=\mathbf{G}_{1}^{T} \mathbf{G}_{13}^{T} \mathbf{M}^{F 1} \mathbf{G}_{12}, \quad \mathbf{M}_{13}=\mathbf{G}_{2}^{T} \mathbf{G}_{23}^{T} \mathbf{M}^{F 2} \mathbf{G}_{22}, \quad \mathbf{m}_{14}=\mathbf{G}_{1}^{T} \mathbf{G}_{13}^{T} \mathbf{M}^{F 1} \mathbf{G}_{13} \mathbf{s}_{1}, \\
& \mathbf{m}_{15}=\mathbf{G}_{2}^{T} \mathbf{G}_{23}^{T} \mathbf{M}^{F 2} \mathbf{G}_{23} \mathbf{S}_{2}, \quad \mathbf{m}_{16}=\mathbf{G}_{1}^{T} \mathbf{G}_{13}^{T} \mathbf{M}^{F 1} \mathbf{G}_{12} \mathbf{s}_{1}, \quad \mathbf{m}_{17}=\mathbf{G}_{2}^{T} \mathbf{G}_{23}^{T} \mathbf{M}^{F 2} \mathbf{G}_{22} \mathbf{S}_{2}, \\
& \mathbf{m}_{18}=\mathbf{G}_{1}^{T} \mathbf{G}_{13}^{T} \mathbf{M}^{F 1} \mathbf{G}_{13} \mathbf{s}_{H 1}+\mathbf{G}_{1}^{T} \mathbf{M}^{N 1} \mathbf{s}_{H 1}, \quad \mathbf{m}_{19}=\mathbf{G}_{2}^{T} \mathbf{G}_{23}^{T} \mathbf{M}^{F 2} \mathbf{G}_{23} \mathbf{s}_{H 2}+\mathbf{G}_{2}^{T} \mathbf{M}^{N 2} \mathbf{s}_{H 2}, \\
& \mathbf{M}_{22}=\mathbf{G}_{11}^{T} \mathbf{M}^{A 1} \mathbf{G}_{11}+\mathbf{G}_{12}^{T} \mathbf{M}^{F 1} \mathbf{G}_{12}, \quad \mathbf{m}_{24}=\mathbf{G}_{12}^{T} \mathbf{M}^{F 1} \mathbf{G}_{13} \mathbf{s}_{1}, \\
& \mathbf{m}_{26}=\mathbf{G}_{12}^{T} \mathbf{M}^{F 1} \mathbf{G}_{12} \mathbf{S}_{1}, \quad \mathbf{m}_{28}=\mathbf{G}_{12}^{T} \mathbf{M}^{F 1} \mathbf{G}_{13} \mathbf{S}_{H 1}, \quad \mathbf{M}_{33}=\mathbf{G}_{21}^{T} \mathbf{M}^{A 2} \mathbf{G}_{21}+\mathbf{G}_{22}^{T} \mathbf{M}^{F 2} \mathbf{G}_{22}, \\
& \mathbf{m}_{35}=\mathbf{G}_{22}^{T} \mathbf{M}^{F 2} \mathbf{G}_{23} \mathbf{s}_{2}, \quad \mathbf{m}_{37}=\mathbf{G}_{22}^{T} \mathbf{M}^{F 2} \mathbf{G}_{22} \mathbf{S}_{2}, \quad \mathbf{m}_{39}=\mathbf{G}_{22}^{T} \mathbf{M}^{F 2} \mathbf{G}_{23} \mathbf{s}_{H 2}, \\
& m_{44}=\mathbf{s}_{1}^{T} \mathbf{G}_{13}^{T} \mathbf{M}^{F 1} \mathbf{G}_{13} \mathbf{s}_{1}, \quad m_{46}=\mathbf{s}_{1}^{T} \mathbf{G}_{13}^{T} \mathbf{M}^{F 1} \mathbf{G}_{12} \mathbf{s}_{1}, \quad m_{48}=\mathbf{s}_{H 1}^{T} \mathbf{G}_{13}^{T} \mathbf{M}^{F 1} \mathbf{G}_{13} \mathbf{s}_{1}, \\
& m_{55}=\mathbf{s}_{2}^{T} \mathbf{G}_{23}^{T} \mathbf{M}^{F 2} \mathbf{G}_{23} \mathbf{s}_{2}, \quad m_{57}=\mathbf{s}_{2}^{T} \mathbf{G}_{23}^{T} \mathbf{M}^{F 2} \mathbf{G}_{22} \mathbf{s}_{2}, \quad m_{59}=\mathbf{s}_{H 2}^{T} \mathbf{G}_{23}^{T} \mathbf{M}^{F 2} \mathbf{G}_{23} \mathbf{s}_{2}, \\
& m_{66}=\mathbf{s}_{1}^{T} \mathbf{G}_{12}^{T} \mathbf{M}^{F 1} \mathbf{G}_{12} \mathbf{s}_{1}, \quad m_{68}=\mathbf{s}_{H 1}^{T} \mathbf{G}_{13}^{T} \mathbf{M}^{F 1} \mathbf{G}_{12} \mathbf{s}_{1}, \quad m_{77}=\mathbf{s}_{2}^{T} \mathbf{G}_{22}^{T} \mathbf{M}^{F 2} \mathbf{G}_{22} \mathbf{s}_{2}, \\
& m_{79}=\mathbf{s}_{H 2}^{T} \mathbf{G}_{23}^{T} \mathbf{M}^{F 2} \mathbf{G}_{22} \mathbf{S}_{2}, \quad m_{88}=\mathbf{s}_{H 1}^{T} \mathbf{G}_{13}^{T} \mathbf{M}^{F 1} \mathbf{G}_{13} \mathbf{s}_{H 1}+\mathbf{s}_{H 1}^{T} \mathbf{M}^{N 1} \mathbf{s}_{H 1}, \\
& m_{99}=\mathbf{s}_{H 2}^{T} \mathbf{G}_{23}^{T} \mathbf{M}^{F 2} \mathbf{G}_{23} \mathbf{S}_{H 2}+\mathbf{s}_{H 2}^{T} \mathbf{M}^{N 2} \mathbf{s}_{H 2}
\end{aligned}
$$


$R_{J 1}, R_{J 2}$ and $H_{J}$ being passive joints, the angular momentum acting on them vanishes, i.e. $p_{i j}=0$. The Cartesian mass matrix $\mathbf{M}_{e} \in \mathbf{R}^{6 \times 6}$ maps the small-amplitude twist of the Peppermill into the momentum applied onto it, namely $\mathbf{p}_{J}=\mathbf{0}$. From eq. (31), the Cartesian mass matrix is obtained:

$$
\mathbf{M}_{e}=\mathbf{M}_{11}-\mathbf{M}_{b 1} \mathbf{M}_{b 2}^{-1} \mathbf{M}_{b 1}^{T}
$$

where

$$
\left.\mathbf{M}_{b 1}=\left[\begin{array}{l}
\mathbf{M}_{12}^{T} \\
\mathbf{M}_{13}^{T} \\
\mathbf{m}_{14}^{T} \\
\mathbf{m}_{15}^{T} \\
\mathbf{m}_{16}^{T} \\
\mathbf{m}_{17}^{T} \\
\mathbf{m}_{18}^{T} \\
\mathbf{m}_{19}^{T}
\end{array}\right]^{T}\right]^{T}, \quad \mathbf{M}_{b 2}=\left[\begin{array}{cccccccc}
\mathbf{M}_{22} & \mathbf{0} & \mathbf{m}_{24} & \mathbf{0} & \mathbf{m}_{26} & \mathbf{0} & \mathbf{m}_{28} & \mathbf{0} \\
\mathbf{O} & \mathbf{M}_{33} & \mathbf{0} & \mathbf{m}_{35} & \mathbf{0} & \mathbf{m}_{37} & \mathbf{0} & \mathbf{m}_{39} \\
\mathbf{m}_{24}^{T} & \mathbf{0}^{T} & m_{44} & 0 & m_{46} & 0 & m_{48} & 0 \\
\mathbf{0}^{T} & \mathbf{m}_{35}^{T} & 0 & m_{55} & 0 & m_{57} & 0 & m_{59} \\
\mathbf{m}_{26}^{T} & \mathbf{0}^{T} & m_{46} & 0 & m_{66} & 0 & m_{68} & 0 \\
\mathbf{0}^{T} & \mathbf{m}_{37}^{T} & 0 & m_{57} & 0 & m_{77} & 0 & m_{79} \\
\mathbf{m}_{28}^{T} & \mathbf{0}^{T} & m_{48} & 0 & m_{68} & 0 & m_{88} & 0 \\
\mathbf{0}^{T} & \mathbf{m}_{39}^{T} & 0 & m_{59} & 0 & m_{79} & 0 & m_{99}
\end{array}\right]
$$

\section{THE TEST TRAJECTORY}

In order to measure the speed of a pick-and-place robot, a standard industrial test cycle has been defined. The original (non-smooth) cycle is known as the Adept test cycle. This trajectory involves a vertical upward translation of $25 \mathrm{~mm}$, a horizontal translation of $300 \mathrm{~mm}$ and a final vertical downward translation of 25 $\mathrm{mm}$. The MP has to move through this trajectory back and forth with a rotation of $180^{\circ}$ during the horizontal segment. The MP is at rest at the initial and final locations, the two other locations being intermediate points. The motion between these points takes place along a straight line. Gauthier et al. (Gauthier et al. 2008) proposed a smooth blending of the non-smooth Adept curve using cubic Lamé curves and an optimum selection of the blending points on the vertical and horizontal segments. This trajectory is the one used in this paper. For the record, the Quattro robot is capable of three cycles per second.

\section{FOURIER ANALYSIS}

It is essential to obtain the frequency spectrum of a highly repetitive mechanical system because the natural frequencies should be placed outside of it to avoid resonance. The frequency spectrum is obtained 
by means of Fourier analysis. A periodic function $f(t)$ with a fundamental frequency $\omega$ can be represented as:

$$
f(t)=\frac{a_{0}}{2}+\sum_{i=1}^{\infty} a_{i} \cos (i \omega t)+\sum_{i=1}^{\infty} b_{i} \sin (i \omega t)
$$

where

$$
a_{0}=\frac{1}{T} \int_{-T / 2}^{T / 2} f(t) d t, a_{i}=\frac{1}{T} \int_{-T / 2}^{T / 2} f(t) \cos (i \omega t) d t, b_{i}=\frac{1}{T} \int_{-T / 2}^{T / 2} f(t) \sin (i \omega t) d t
$$

in which $i$ and $T$ are the harmonic index and the period of function $f(t)$, respectively. In this case, the periodic functions are the trajectory functions of the moving platform (MP), namely, the translations along the $x$-, the $y$ - and the $z$-axes along with the rotation about the $z$-axis.

In order to obtain the excitation frequency spectrum and find the highest operation speed whose excitation frequency spectrum is under the first natural frequency of the PMC, the distribution of normalized parameters $\left|\bar{a}_{x n}\right|,\left|\bar{a}_{y n}\right|,\left|\bar{a}_{z n}\right|$ and $\left|\bar{a}_{\phi n}\right|$ with respect to the frequency $f$ when the operation frequency is 1 and 2 cycles/s are plotted in Figs. 7 and 8, respectively.
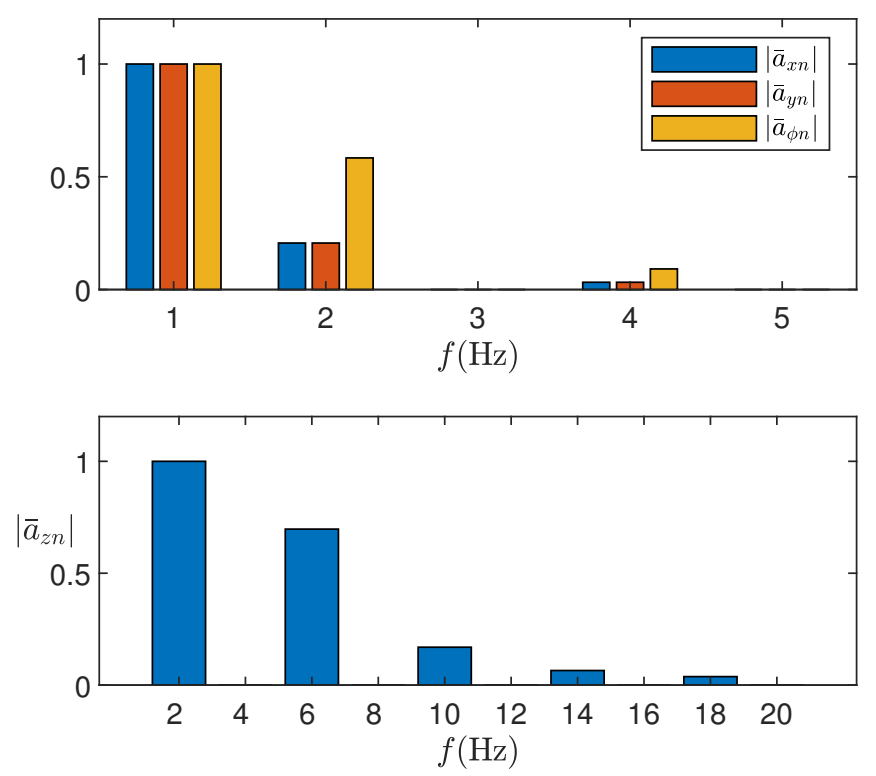

Fig. 7. Amplitudes of the harmonics of the four independent motions vs. natural frequencies (for an operation frequency of 1 cycle/s): the frequency of the cyclical translation along the $z$-axis is twice as large as the three other independent motions 

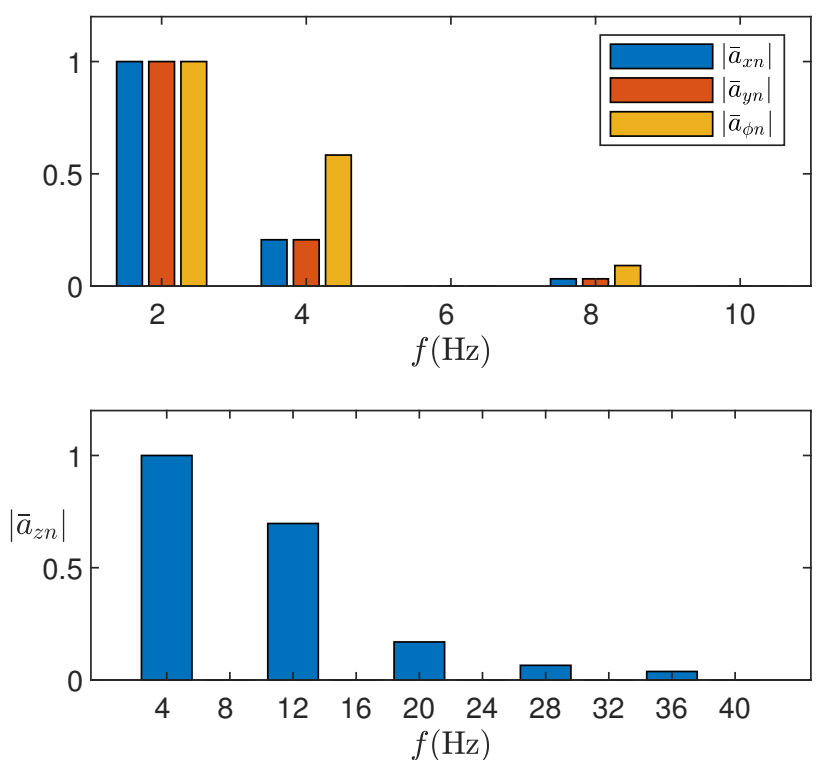

Fig. 8. Amplitudes of the harmonics of the four independent motions vs. natural frequencies (for an operation frequency of 2 cycles/s): the frequency of the cyclical translation along the $z$-axis is twice as large as the three other independent motions

Modal analysis calls for the stiffness and mass matrices, obtained above. The mathematical model of the robot, at an equilibrium posture, is

$$
\mathbf{M} \ddot{\mathbf{x}}+\mathbf{K} \dot{\mathbf{x}}=\mathbf{0}
$$

where $\mathbf{M}$ and $\mathbf{K}$ are the Cartesian mass and stiffness matrices, $\mathbf{x}$ the SAD screw. To obtain the natural frequencies of the system, the well-known dynamic-matrix can be used. One has

$$
(\lambda \mathbf{M}+\mathbf{K}) \mathbf{u}=\mathbf{0}
$$

where $\lambda$ and $\mathbf{u}$ are, respectively, the eigenvalue and the corresponding eigenvector of the above eigenvalue problem. Therefore, by computing the eigenvalue of $\mathbf{M}^{-1} \mathbf{K}$, the set of values $-\omega^{2}$ is obtained, with $\omega$ denoting one of the natural frequencies. It should be noted, however, that the last three components of the six-dimensional eigenvector $\mathbf{u}$ carry units of length, while the first three are dimensionless.

With the stiffness $\left(\mathbf{K}_{e}\right)$ and mass $\left(\mathbf{M}_{e}\right)$ matrices, respectively obtained in Subsection 2.2 and Section 3, the natural frequencies along the Adept test cycle were obtained, as displayed in Fig. 9. Only the first natural frequency is shown, as the subsequent frequencies are well above the first, and thus not significant.

As per Fig. 9, the minimum value of the first natural frequency is $52.5 \mathrm{~Hz}$. According to Fig. 8, the 


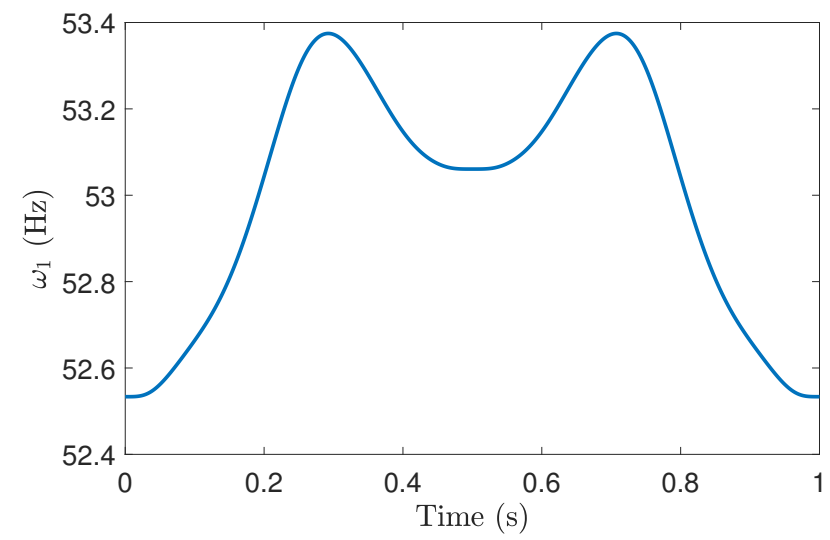

Fig. 9. The evolution of the first natural frequency of the PMC along the test trajectory $\omega_{1}(\mathrm{~Hz})$

excitation frequency spectrum for an operation frequency of 2 cycles/s, we can see that the translation along the $x$ - and $y$-axis and rotation about the $z$-axis are obviously on the safe side. The translation along the $z$-axis has already reached the limit; hence, resonance will ensue for operation frequencies above this threshold.

\section{CONCLUSIONS}

The main challenge faced by pick-and-place robots is speed. High-speeds are prone to lead to resonance, which calls for an elastodynamics analysis of the robot to verify that the harmonics of the prescribed trajectory do not lie within the frequency spectrum of the robot structure. The industry standard Adept test cycle was used to obtain numerical data of the frequency content of the cycle at different frequencies. The model and analysis proposed in this paper show that operation frequencies under 3 cycles per second are not problematic. For higher operation frequencies, the current structural design of the robot under development, the PMC, will have to be revised.

\section{REFERENCES}

Al-Widyan, K. and Angeles, J. 2004. The robust design of Schönflies-motion generators. In On Advances in Robot Kinematics. Springer. pp. 339-350.

Angeles, J. 2004. The qualitative synthesis of parallel manipulators. Transactions of the ASME-Journal of Mechanical Design. 126(4): 617-624.

Belzile, B. and Angeles, J. 2019. Heuristic Algorithm for Velocity Scheduling with a Schönflies-Motion Generator.

In T. Uhl, editor, Advances in Mechanism and Machine Science. IFToMM WC 2019. Mechanisms and Machine Science, vol. 73. Springer, Cham. pp. 2411-2419. 
Belzile, B., Karimi Eskandary, P., and Angeles, J. 2020. Workspace Determination and Feedback Control of a Pickand-Place Parallel Robot: Analysis and Experiments. IEEE Robotics and Automation Letters. 5(1): 40-47.

Carricato, M. 2005. Fully isotropic four-degrees-of-freedom parallel mechanisms for Schönflies motion. The International Journal of Robotics Research. 24(5): 397-414.

Company, O., Krut, S., and Pierrot, F. 2002. Modelling and preliminary design issues of a four-axis parallel machine for heavy parts handling. Proceedings of the Institution of Mechanical Engineers, Part K: Journal of Multi-body Dynamics. 216(1): 1-12.

Dubowsky, S. 1994. Dealing with vibrations in the deployment structures of space robotic systems. In Proceedings of the 5th International Conference on Adaptive Structures. Sendai, Japan. pp. 5-7.

Frisoli, A., Checcacci, D., Salsedo, F., and Bergamasco, M. 2000. Synthesis by screw algebra of translating in-parallel actuated mechanisms. In Advances in robot kinematics. Springer. pp. 433-440.

Gauthier, J.-F., Angeles, J., and Nokleby, S. 2008. Optimization of a test trajectory for SCARA systems. In Advances in Robot Kinematics: Analysis and Design. Springer. pp. 225-234.

Gauthier, J.-F., Angeles, J., Nokleby, S. B., and Morozov, A. 2009. The kinetostatic conditioning of two-limb Schönflies motion generators. Journal of Mechanisms and Robotics. 1(1): 011010.

Gogu, G. 2004. Fully-isotropic T3R1-type parallel manipulators. In On Advances in Robot Kinematics. Springer. pp. $265-272$.

Gogu, G. 2005. Singularity-free fully-isotropic parallel manipulators with Schönflies motions. In Proceedings of the 12th International Conference on Advanced Robotics, 2005. IEEE, Seattle, Wash., USA. pp. 194-201.

Gogu, G. 2007. Structural synthesis of fully-isotropic parallel robots with Schönflies-motions via theory of linear transformations and evolutionary morphology. European Journal of Mechanics-A/Solids. 26(2): 242-269.

Harada, T. and Angeles, J. 2014. Kinematics and singularity analysis of a CRRHHRRC parallel Schönflies motion generator. CSME Trans. 38(2): 173-183.

Harada, T., Friedlaender, T., and Angeles, J. 2014. The development of an innovative two-DOF cylindrical drive: Design, analysis and preliminary tests. In Proceedings of the 2014 IEEE International Conference on Robotics and Automation. Hong Kong. pp. 6338-6344.

Hervé, J. M. 2004. New translational parallel manipulators with extensible parallelogram. In Proceedings of the 11th World Congress in Mechanism and Machine Science. volume 4. IFToMM. pp. 1599-1603.

Karimi Eskandary, P. and Angeles, J. 2018. The translating П-joint: design and applications. Mechanism and Machine Theory. 122: 361-370.

Karimi Eskandary, P., Belzile, B., and Angeles, J. 2019. Trajectory-Planning and Normalized-Variable Control for Parallel Pick-and-Place Robots. Journal of Mechanisms and Robotics. 11(3): 031001-031001-8. doi: $10.1115 / 1.4042631$. 
Kong, X. and Gosselin, C. M. 2004. Type synthesis of 3-DOF translational parallel manipulators based on screw theory. ASME. J. Mech. Des. 126(1): 83-92.

Krut, S., Company, O., Nabat, V., and Pierrot, F. 2006. Heli4: a parallel robot for SCARA motions with a very compact traveling plate and a symmetrical design. In Proceedings of the 2006 IEEE/RSJ International Conference on Intelligent Robots and Systems. IEEE, Beijing, China. pp. 1656-1661.

Krut, S., Nabat, V., Company, O., and Pierrot, F. 2004. A high-speed parallel robot for SCARA motions. In Proceedings of the IEEE International Conference on Robotics and Automation. volume 4. IEEE, New Orleans, LA, USA. pp. 4109-4115.

Kumar, R., Berkelman, P., Gupta, P., Barnes, A., Jensen, P. S., Whitcomb, L. L., and Taylor, R. H. 2000. Preliminary experiments in cooperative human/robot force control for robot assisted microsurgical manipulation. In Proceedings of the 2000 IEEE International Conference on Robotics and Automation. volume 1. IEEE. pp. 610-617.

Lee, P.-C. and Lee, J.-J. 2012. Singularity and workspace analysis of three isoconstrained parallel manipulators with schoenflies motion. Frontiers of Mechanical Engineering. 7(2): 163-187.

Lee, P.-C. and Lee, J.-J. 2016. On the kinematics of a new parallel mechanism with Schönfliesmotion. Robotica. 34(09): 2056-2070.

Lee, P.-C., Lee, J.-J., and Lee, C.-C. 2010. Four novel pick-and-place isoconstrained manipulators and their inverse kinematics. In Proceedings of the ASME International Design Engineering Technical Conferences and Computers and Information in Engineering Conference. American Society of Mechanical Engineers, Montreal, Canada. pp. 1079-1088.

Li, Q., Huang, Z., and Hervé, J. M. 2004. Type synthesis of 3R2T 5-DOF parallel mechanisms using the Lie group of displacements. IEEE transactions on robotics and automation. 20(2): 173-180.

Lončarić, J. 1985. Geometrical Analysis of Compliant Mechanisms in Robotics (Euclidean Group, Elastic Systems, Generalized Springs. Ph.D. thesis. Cambridge, MA, USA. AAI8520241.

Makino, H., Kato, A., and Yamazaki, Y. 2007. Research and commercialization of SCARA Robot-The case of industry-university joint research and development. International Journal of Automation Technology. 1: 61-67.

Meggiolaro, M. A. and Dubowsky, S. 2001. Improving the positioning accuracy of powerful manipulators with application in nuclear maintenance. In Proceedings of the 16th Brazilian Congress of Mechanical Engineering on Robotics and Control. volume 15. ABCM, Uberlândia, Minas Gerais, Brazil. pp. 210-219.

Mises, R. V. 1924. Motorrechnung, ein neues Hilfsmittel der Mechanik. ZAMM - Journal of Applied Mathematics and Mechanics / Zeitschrift für Angewandte Mathematik und Mechanik. 4(2): 155-181.

Nabat, V., Rodriguez, M., Company, O., Krut, S., and Pierrot, F. 2005. Par4: Very high speed parallel robot for pick-and-place. In Proceedings of the 2005 IEEE/RSJ International Conference on Intelligent Robots and Systems. IEEE, Edmonton, Alberta, Canada. pp. 553-558. 
Pierrot, F. and Company, O. 1999. H4: A new family of 4-DOF parallel robots. In Proceedings of the 1999 IEEE/ASME International Conference on Advanced Intelligent Mechatronics. IEEE, Atlanta, GA, USA. pp. 508513.

Pierrot, F., Nabat, V., Company, O., Krut, S., and Poignet, P. 2009. Optimal design of a 4-DOF parallel manipulator: From academia to industry. IEEE Transactions on Robotics. 25(2): 213-224.

Pierrot, F., Shibukawa, T., Morita, K., et al. 2003. Four-degree-of-freedom parallel robot. US Patent 6,516,681.

Pradeep, A., Yoder, P. J., and Mukundan, R. 1989. On the Use of Dual- Matrix Exponentials in Robotic Kinematics. The International Journal of Robotics Research. 8(5): 57-66.

Salisbury, J. K. and Craig, J. J. 1982. Articulated hands: Force control and kinematic issues. The International journal of Robotics research. 1(1): 4-17.

Schoenflies, A. 1893. La géométrie du mouvement: exposé synthétique. Gauthier-Villars et fils.

Shaheed, M., Poerwanto, H., and Tokhi, M. 2005. Adaptive inverse-dynamic and neuro-inverse-dynamic active vibration control of a single-link flexible manipulator. Proceedings of the Institution of Mechanical Engineers, Part I: Journal of Systems and Control Engineering. 219(6): 431-448.

Yamano, M., Konno, A., and Uchiyama, M. 2000. Experiments on capturing a floating object by two flexible manipulators. In Proceedings of the 2000 IEEE International Conference on Robotics and Automation. volume 1. IEEE, San Francisco, CA, USA. pp. 482-487.

Yin, Z., Belzile, B., Angeles, J., and Forbes, J. R. 2019. Elastodynamics of a Parallel Schonfliës-motion generator. In Proceedings of the 2019 CCToMM Symposium on Mechanisms, Machines, and Mechatronics. CCToMM, Montreal, Canada. pp. 1-12, paper 21.

Zhang, Q., Li, C., Zhang, J., and Jin, J. 2016. Active Vibration Control and Coupled Vibration Analysis of a Parallel Manipulator with Multiple Flexible Links. Shock and Vibration. 2016. 


\section{LIST OF FIGURES}

$1 \quad$ Kinematics of the $\mathrm{PMC} \ldots \ldots \ldots \ldots \ldots \ldots$

2 Elastostatics of the $\mathrm{PMC} \ldots \ldots \ldots \ldots \ldots \ldots$

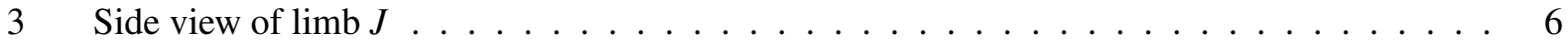

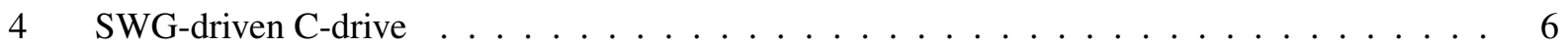

5 The concept of Cartesian spring: (a) two rigid plates coupled by a Cartesian spring; (b) the coupling of two Cartesian springs via a $\mathrm{R}$ joint $\ldots \ldots \ldots \ldots$

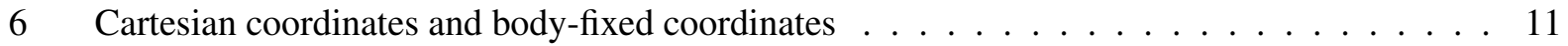

7 Amplitudes of the harmonics of the four independent motions vs. natural frequencies (for an operation frequency of $1 \mathrm{cycle} / \mathrm{s})$ : the frequency of the cyclical translation along the $z$-axis is twice as large as the three other independent motions . . . . . . . . . . . . . 16

8 Amplitudes of the harmonics of the four independent motions vs. natural frequencies (for an operation frequency of 2 cycles/s): the frequency of the cyclical translation along the $z$-axis is twice as large as the three other independent motions $\ldots \ldots \ldots \ldots \ldots$

9 The evolution of the first natural frequency of the PMC along the test trajectory $\omega_{1}(\mathrm{~Hz}) \ldots 18$ 